PROCEEDINGS OF THE

AMERICAN MATHEMATICAL SOCIETY

Volume 128, Number 8, Pages 2261-2270

S 0002-9939(99)05278-8

Article electronically published on November 29, 1999

\title{
FROBENIUS PROPERTIES AND MASCHKE-TYPE THEOREMS FOR ENTWINED MODULES
}

\author{
TOMASZ BRZEZIŃSKI
}

(Communicated by Ken Goodearl)

\begin{abstract}
Entwined modules arose from the coalgebra-Galois theory. They are a generalisation of unified Doi-Hopf modules. In this paper, Frobenius properties and Maschke-type theorems known for Doi-Hopf modules are extended to the case of entwined modules.
\end{abstract}

\section{INTRODUCTION}

The aim of this article is to show that results of recent papers [6] and [7] concerning Frobenius properties and a Maschke-type theorem for Doi-Hopf modules [9], 11] hold for the more general class of modules, known as entwined modules [3]. These are modules of an algebra and comodules of a coalgebra such that the action and the coaction satisfy a certain compatibility condition. Unlike Doi-Hopf modules, entwined modules are defined purely using the properties of an algebra and a coalgebra combined into an entwining structure. There is no need for a "background" bialgebra, which is an indispensable part of the Doi-Hopf construction. The bialgebra-free formulation - apart from being more general - also has a remarkable self-duality property, which essentially implies that for every statement involving module structure of an entwined module there is a corresponding statement involving its comodule structure. As an illustration of this phenomenon we give two Maschke-type theorems for entwined modules.

The paper is organised as follows. In Section 2 we recall definitions and give examples of entwining structures and entwined modules. In Section 3 we introduce integrals for entwining structures and analyse Frobenius properties of entwined modules generalising the results of [6]. Finally, in Section 4 we state a Maschketype theorem for entwined modules and derive its dual form.

We work over a commutative ring $k$. We assume that all the algebras are over $k$ and unital, and the coalgebras are over $k$ and counital. The unadorned tensor product is over $k$. For any $k$-modules $V, W$ the $\operatorname{symbol} \operatorname{Hom}(V, W)$ denotes the $k$-module of $k$-module maps $V \rightarrow W$, the identity map $V \rightarrow V$ is denoted by $V, V^{*}=\operatorname{Hom}(V, k)$, and $\mathrm{ev}_{V}: V \otimes V^{*} \rightarrow k$ denotes the evaluation map, i.e. $\operatorname{ev}_{V}: v \otimes v^{*} \mapsto\left\langle v, v^{*}\right\rangle=v^{*}(v)$. We also implicitly identify $V$ with $V \otimes k$ and $k \otimes V$ via the canonical isomorphisms.

Received by the editors June 16, 1998 and, in revised form, September 21, 1998.

1991 Mathematics Subject Classification. Primary 16W30, 16W35, 16 S40.

The author is a Lloyd's of London Tercentenary Fellow. 
For a $k$-algebra $A$ we use $\mu$ to denote the product as a map and $1_{A}$ to denote the unit both as an element of $A$ and as a map $k \rightarrow A, \alpha \rightarrow \alpha 1_{A}$. $\mathbf{M}_{A}$ (resp. $\left.{ }_{A} \mathbf{M}\right)$ denotes the category of right (resp. left) $A$-modules. The morphisms in this category are denoted by $\operatorname{Hom}_{A}(M, N)\left(\operatorname{resp} \cdot{ }_{A} \operatorname{Hom}(M, N)\right)$. For any $M \in \mathbf{M}_{A}$ (resp. $M \in{ }_{A} \mathbf{M}$ ), the symbol $\rho_{M}$ (resp. ${ }_{M} \rho$ ) denotes the action as a map (on elements the action is denoted by a dot). We often write $M_{A}$ (resp. $\left.{ }_{A} M\right)$ to indicate in which context the $A$-module $M$ appears. If $A, B$ are $k$-algebras and $M, N$ are $(A, B)$-bimodules, then ${ }_{A} \operatorname{Hom}_{B}(M, N)$ denotes the set of $(A, B)$-bimodule maps.

For a $k$-coalgebra $C$ we use $\Delta$ to denote the coproduct and $\epsilon$ to denote the counit. Notation for comodules is similar to that for modules but with subscripts replaced by superscripts, i.e. $\mathbf{M}^{C}$ is the category of right $C$-comodules, $\rho^{M}$ is a right coaction etc. We use the Sweedler notation for coproducts and coactions, i.e. $\Delta(c)=c_{(1)} \otimes c_{(2)}, \rho^{M}(m)=m_{(0)} \otimes m_{(1)}$ (summation understood). The symbol $\triangleright$ (resp. $\triangleleft$ ) stands for the standard left (resp. right) action of the (convolution product) algebra $C^{*}$ on $C$ induced by the coproduct in $C$.

\section{Preliminaries on Entwining Structures}

Definition 2.1. An entwining structure (over $k$ ) is a triple $(A, C)_{\psi}$ consisting of a $k$-algebra $A$, a $k$-coalgebra $C$ and a $k$-module map $\psi: C \otimes A \rightarrow A \otimes C$ satisfying

$$
\begin{array}{cc}
\psi \circ(C \otimes \mu)=(\mu \otimes C) \circ(A \otimes \psi) \circ(\psi \otimes A), & \psi \circ\left(C \otimes 1_{A}\right)=1_{A} \otimes C, \\
(A \otimes \Delta) \circ \psi=(\psi \otimes C) \circ(C \otimes \psi) \circ(\Delta \otimes A), & (A \otimes \epsilon) \circ \psi=\epsilon \otimes A .
\end{array}
$$

A morphism of entwining structures is a pair $(f, g):(A, C)_{\psi} \rightarrow(\tilde{A}, \tilde{C})_{\tilde{\psi}}$, where $f: A \rightarrow \tilde{A}$ is an algebra map, $g: C \rightarrow \tilde{C}$ is a coalgebra map, and $(f \otimes g) \circ \psi=$ $\tilde{\psi} \circ(g \otimes f)$.

For $(A, C)_{\psi}$ we use the notation $\psi(c \otimes a)=a_{\alpha} \otimes c^{\alpha}$ (summation over a Greek index understood), for all $a \in A, c \in C$. The notion of an entwining structure was introduced in [5] Definition 2.1]. It is self-dual in the sense that conditions in Definition 2.1 are invariant under the operation consisting of interchanging $A$ with $C, \mu$ with $\Delta$, and $1_{A}$ with $\epsilon$, and reversing the order of maps. Below are two main classes of examples of entwining structures.

Example 2.2. Let $H$ be a bialgebra, $C$ a right $H$-module coalgebra, and $A$ a right $H$-comodule algebra. Then $C$ and $A$ are entwined by $\psi: C \otimes A \rightarrow A \otimes C$, $c \otimes a \mapsto a_{(0)} \otimes c \cdot a_{(1)}$. The corresponding entwining structure $(A, C)_{\psi}$ is called an entwining structure associated to a Doi-Hopf datum $(A, C, H)$. Note that if $H$ has a bijective antipode $S$, then $\psi$ is bijective with $\psi^{-1}: a \otimes c \mapsto c \cdot S^{-1} a_{(1)} \otimes a_{(0)}$.

Example 2.3 (4). Let $C$ be a coalgebra, $A$ an algebra and a right $C$-comodule. Let $B:=\left\{b \in A \mid \forall a \in A, \rho^{A}(b a)=b \rho^{A}(a)\right\}$ and assume that the canonical left $A$ module, right $C$-comodule map can $: A \otimes_{B} A \rightarrow A \otimes C, a \otimes a^{\prime} \mapsto a \rho^{A}\left(a^{\prime}\right)$, is bijective. Let $\psi: C \otimes A \rightarrow A \otimes C$ be a $k$-linear map given by $\psi(c \otimes a)=\operatorname{can}\left(\operatorname{can}^{-1}\left(1_{A} \otimes c\right) a\right)$. Then $(A, C)_{\psi}$ is an entwining structure. The extension $B \subset A$ is called a coalgebraGalois extension (or a $C$-Galois extension) and is denoted by $A(B)^{C} .(A, C)_{\psi}$ is the canonical entwining structure associated to $A(B)^{C}$.

Definition 2.4. Let $(A, C)_{\psi}$ be an entwining structure. An (entwined) $(A, C)_{\psi}$ module is a right $A$-module, right $C$-comodule $M$ such that

$$
\rho^{M} \circ \rho_{M}=\left(\rho_{M} \otimes C\right) \circ(M \otimes \psi) \circ\left(\rho^{M} \otimes A\right)
$$


(explicitly: $\left.\rho^{M}(m \cdot a)=m_{(0)} \cdot a_{\alpha} \otimes m_{(1)}^{\alpha}, \forall a \in A, m \in M\right)$. A morphism of $(A, C)_{\psi^{-}}$ modules is a right $A$-module map which is also a right $C$-comodule map. The category of $(A, C)_{\psi}$-modules is denoted by $\mathbf{M}_{A}^{C}(\psi)$.

The category $\mathbf{M}_{A}^{C}(\psi)$ was introduced and studied in [3]. Modules associated to the entwining structure in Example 2.2 are unifying Hopf modules or Doi-Hopf modules introduced in [9], [11]. On the other hand, entwined modules associated to the entwining structure in Example 2.3 do not seem to be of Doi-Hopf type. The following example is a special case of the construction in [3. Section 3].

Example 2.5. Let $(A, C)_{\psi}$ be an entwining structure. Then

(1) If $M$ is a right $A$-module, then $M \otimes C \in \mathbf{M}_{A}^{C}(\psi)$ with the coaction $M \otimes \Delta$ and the action $(m \otimes c) \cdot a=m \cdot \psi(c \otimes a)$, for all $a \in A, c \in C$ and $m \in M$. In particular $A \otimes C \in \mathbf{M}_{A}^{C}(\psi)$.

(2) If $V$ is a right $C$-comodule, then $V \otimes A \in \mathbf{M}_{A}^{C}(\psi)$ with the action $V \otimes \mu$ and the coaction $v \otimes a \mapsto v_{(0)} \otimes \psi\left(v_{(1)} \otimes a\right)$ for any $a \in A$ and $v \in V$. In particular $C \otimes A \in \mathbf{M}_{A}^{C}(\psi)$.

\section{Integrals AND Frobenius PROPERTIES OF ENTWINED MOdUles}

An entwining structure $(A, C)_{\psi}$ is said to be factorisable if there exists a unique map $\bar{\psi}: A \otimes C^{*} \rightarrow C^{*} \otimes A$ such that the following diagram

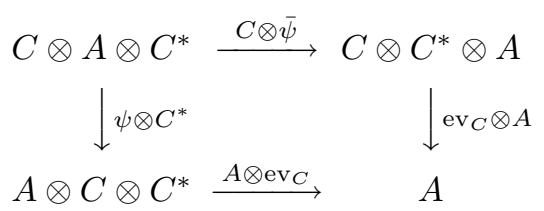

commutes. For any $a \in A$ and $\xi \in C^{*}$ we write $\bar{\psi}(a \otimes \xi)=\xi_{i} \otimes a^{i}$ (summation over repeated index understood). For example, $(A, C)_{\psi}$ associated to a Doi-Hopf datum in Example 2.2 is factorisable, provided $C$ is a projective $k$-module. Explicitly, for all $a \in A, \xi \in C^{*}, \bar{\psi}(a \otimes \xi)=a_{(1)} \cdot \xi \otimes a_{(0)}$, where the left action of $H$ on $C^{*}$ is induced from the right action of $H$ on $C$ via $\langle c, h \cdot \xi\rangle=\langle c \cdot h, \xi\rangle$ for all $c \in C, \xi \in C^{*}$ and $h \in H$. Also, any $(A, C)_{\psi}$ with $C$ being a finitely generated projective $k$-module is factorisable. In this case the factorisation is $\psi(a \otimes \xi)=\sum_{n=1}^{N}\left\langle c_{n}{ }^{\alpha}, \xi\right\rangle \xi_{n} \otimes a_{\alpha}$, where $\left\{\xi_{n}, c_{n}\right\}_{n=1}^{N}$ is a dual basis in $C$.

Let $(A, C)_{\psi}$ be a factorisable entwining structure and let $B=C^{* o p}$, i.e. $\left\langle c, b b^{\prime}\right\rangle=$ $\left\langle c_{(2)}, b\right\rangle\left\langle c_{(1)}, b^{\prime}\right\rangle$, for all $b, b^{\prime} \in B, c \in C$. As explained in [5, Proposition 2.7], the triple $(A, B, \bar{\psi})$ defines a factorisation structure (cf. [13 Equations (7.10)]). This allows one to construct a generalised smash product (factorised) algebra $B \#_{\bar{\psi}} A$ on $B \otimes A$ with the product $(b \otimes a)\left(b^{\prime} \otimes a^{\prime}\right)=b \bar{\psi}\left(a \otimes b^{\prime}\right) a^{\prime}$, for all $a, a^{\prime} \in A, b, b^{\prime} \in B$. The maps $A \hookrightarrow B \#_{\bar{\psi}} A, a \mapsto 1_{B} \otimes a$ and $B \hookrightarrow B \#_{\bar{\psi}} A, b \mapsto b \otimes 1_{A}$ are algebra inclusions. Every $B \#_{\bar{\psi}} A$-module is viewed as an $A$ or $B$ module via these maps. Given such $B \#_{\bar{\psi}} A$ we equip the $k$-module $\operatorname{Hom}(B, A)$ with the structure of an $\left(A, B \#_{\bar{\psi}} A\right)$-bimodule via $\left(a \cdot f \cdot\left(b \otimes a^{\prime}\right)\right)\left(b^{\prime}\right)=a f\left(b b_{i}^{\prime}\right) a^{\prime i}$, for all $a, a^{\prime} \in A, b, b^{\prime} \in B$, $f \in \operatorname{Hom}(B, A)$. A $k$-module map $\lambda: B \rightarrow A$ such that for all $a \in A, a \cdot \lambda=\lambda \cdot a$, is called an integral in $B \#_{\bar{\psi}} A$. The $k$-module of integrals in $B \#_{\bar{\psi}} A$ is denoted by $\operatorname{Int}\left(B \#_{\bar{\psi}} A\right)$.

Finally, we recall the notion of a Frobenius extension introduced in [10] and [15]. 
Definition 3.1. Let $X$ be an algebra and $A$ its subalgebra. The extension $A \subset X$ is called a Frobenius extension (of the first kind) iff $X$ is a finitely generated projective right $A$-module and $X \cong \operatorname{Hom}_{A}(X, A)$ as $(A, X)$-bimodules. The $(A, X)$-bimodule structure of $\operatorname{Hom}_{A}(X, A)$ is given by $(a \cdot f \cdot x)\left(x^{\prime}\right)=a f\left(x x^{\prime}\right)$, for all $a \in A, x, x^{\prime} \in X$ and $f \in \operatorname{Hom}_{A}(X, A)$.

Proposition 3.2. Let $(A, C)_{\psi}$ be an entwining structure and let $B=C^{* o p}$. Assume that $B$ is a finitely generated projective $k$-module (hence $(A, C)_{\psi}$ is factorisable) and $A$ is a faithfully flat $k$-module, and let $X=B \#_{\bar{\psi}} A$. Then the following are equivalent:

(1) The functor $\operatorname{Hom}(B,-): \mathbf{M}_{A} \rightarrow \mathbf{M}_{X}$ is the left adjoint of the functor $\mathcal{F}: \mathbf{M}_{X} \rightarrow \mathbf{M}_{A}$ induced by $A \hookrightarrow X$.

(2) The extension $A \subset X$ is Frobenius.

(3) $X$ is isomorphic to $\operatorname{Hom}(B, A)$ as an $(A, X)$-bimodule.

(4) There exists an integral $\lambda \in \operatorname{Int}\left(B \#_{\bar{\psi}} A\right)$ such that the map $\phi: B \otimes A \rightarrow$ $\operatorname{Hom}(B, A), b \otimes a \mapsto \lambda \cdot(b \otimes a)$ is bijective.

Proof. (1) $\Leftrightarrow(2) . \operatorname{Hom}(B,-)$ is the right adjoint of $\mathcal{F}$ since for all $M \in \mathbf{M}_{X}, N \in$ $\mathbf{M}_{A}$ there is a natural isomorphism $\eta_{M, N}: \operatorname{Hom}_{X}(M, \operatorname{Hom}(B, N)) \rightarrow \operatorname{Hom}_{A}(M, N)$, $\eta_{M, N}(f)(m)=f(m)\left(1_{B}\right)$, for all $m \in M$. Its inverse is $\eta_{M, N}^{-1}(g)(m)(b)=g(m \cdot b)$, for all $b \in B, m \in M$. By [14, Theorem 3.15] one thus deduces that $A \subset X$ is Frobenius if and only if $\operatorname{Hom}(B,-)$ is also the left adjoint of $\mathcal{F}$.

$(2) \Leftrightarrow(3)$. By assumption, $B$ is a finitely generated projective $k$-module, $A$ is a faithfully flat $k$-module, and $X \cong B \otimes A$ as a right $A$ module. Thus $X$ is a finitely generated projective right $A$-module (cf. [2 I.3.6 Proposition 12]). Therefore $A \subset X$ is a Frobenius extension if and only if $X \cong \operatorname{Hom}_{A}(X, A)$ as $(A, X)$ bimodules. One easily checks that the map $\eta_{B, A}: \operatorname{Hom}(B, A) \rightarrow \operatorname{Hom}_{A}(X, A)$ given by $\eta_{B, A}(f)(b \otimes a)=f(b) a$ for all $a \in A, b \in B$ is an isomorphism of $(A, X)$ bimodules. Its inverse is $\eta_{B, A}^{-1}(g)(b)=g\left(b \otimes 1_{A}\right)$, for all $b \in B$. Combining the Frobenius isomorphism with $\eta_{B, A}^{-1}$ one obtains the required isomorphism of $(A, X)$ modules.

$(3) \Leftrightarrow(4)$. We will show that the map $\theta: \operatorname{Int}\left(B \#_{\bar{\psi}} A\right) \rightarrow{ }_{A} \operatorname{Hom}_{X}(X, \operatorname{Hom}(B, A))$, given by $\theta(\lambda)(x)=\lambda \cdot x$, for all $x \in X, \lambda \in \operatorname{Int}\left(B \#_{\bar{\psi}} A\right)$ is well-defined and bijective. For any integral $\lambda$, the map $\theta(\lambda)$ is clearly a right $X$-module map. Now take any $x \in X, a \in A$ and compute

$$
\begin{aligned}
(a \cdot \theta(\lambda))(x) & =a \cdot \theta(\lambda)(x)=a \cdot \lambda \cdot x \\
& =(\lambda \cdot a) \cdot x \quad(\lambda \text { is an integral }) \\
& =\theta(\lambda)(a x) .
\end{aligned}
$$

Consider $\tilde{\theta}:{ }_{A} \operatorname{Hom}_{X}(X, \operatorname{Hom}(B, A)) \rightarrow \operatorname{Int}\left(B \#_{\bar{\psi}} A\right), \tilde{\theta}: \phi \mapsto \phi\left(1_{X}\right)$. This map is well-defined since $\phi$ is an $(A, A)$-bimodule map and thus for all $a \in A, a \cdot \phi\left(1_{X}\right)=$ $\phi(a)=\phi\left(1_{X}\right) \cdot a$. An easy calculation shows that $\tilde{\theta}$ is the inverse of $\theta$.

If $(A, C)_{\psi}$ is a factorisable entwining structure, then every $M \in \mathbf{M}_{A}^{C}(\psi)$ can be viewed as an object in $\mathbf{M}_{X}$, where $X=C^{* o p} \#_{\bar{\psi}} A$, via the action $m \cdot(\xi \otimes a)=$ $m_{(0)} \cdot a\left\langle m_{(1)}, \xi\right\rangle$, for all $m \in M, a \in A, \xi \in C^{*}$. If $C$ is a finitely generated projective $k$-module, then every right $X$-module $M$ is an object in $\mathbf{M}_{A}^{C}(\psi)$ with the natural right $A$-action and the coaction given by $m_{(0)}\left\langle m_{(1)}, \xi\right\rangle=m \cdot \xi$ for all $m \in M, \xi \in C^{* o p}$. Therefore, if $C$ is a finitely generated projective $k$-module, then 
$\mathbf{M}_{A}^{C}(\psi) \cong \mathbf{M}_{X}$ (cf. [9, (1.3)]). Using this category isomorphism one can reformulate Proposition 3.2. First, however, we need the following

Definition 3.3. Let $(A, C)_{\psi}$ be an entwining structure. An integral in $(A, C)_{\psi}$ is an element $x=\sum_{i=1}^{n} a_{i} \otimes c_{i} \in A \otimes C$ such that for all $a \in A, a \cdot x=x \cdot a$. The left action is the obvious one, while the right action is as in Example 2.5(1). Explicitly, we require $\sum_{i=1}^{n} a a_{i} \otimes c_{i}=\sum_{i=1}^{n} a_{i} \psi\left(c_{i} \otimes a\right)$.

If $(A, C)_{\psi}$ is an entwining structure of Example 2.2, then $x$ is an integral in $(A, C)_{\psi}$ iff it is an $H$-integral in the sense of [6, Definition 2.1].

Example 3.4. Let $(A, C)_{\psi}$ be the canonical entwining structure associated to a coalgebra-Galois extension $A(B)^{C}$ as in Example 2.3. Then $x=\sum_{i=1}^{n} a_{i} \otimes c_{i}$ is an integral in $(A, C)_{\psi}$ iff for all $a \in A, a \cdot x^{\tau}=x^{\tau} \cdot a$, where $x^{\tau}=\sum_{i=1}^{n} a_{i} c a n^{-1}\left(1 \otimes c_{i}\right) \in$ $A \otimes_{B} A\left(A \otimes_{B} A\right.$ has the obvious ( $\left.A, A\right)$-bimodule structure).

The following proposition is a general $(A, C)_{\psi}$-module version of [6, Theorem 2.4].

Proposition 3.5. Let $(A, C)_{\psi}$ be an entwining structure and let $B=C^{* o p}$. If $A$ is a faithfully flat $k$-module and $C$ is a finitely generated projective $k$-module, then the following are equivalent:

(1) The functor $-\otimes C: \mathbf{M}_{A} \rightarrow \mathbf{M}_{A}^{C}(\psi)$ is the left adjoint of the forgetful functor $\mathbf{M}_{A}^{C}(\psi) \rightarrow \mathbf{M}_{A}$.

(2) The extension $A \subset B \#_{\bar{\psi}} A$ is Frobenius.

(3) $C^{*} \otimes A \cong A \otimes C$ as $\left(A, B \#_{\bar{\psi}} A\right)$-bimodules.

(4) $C^{*} \otimes A \cong A \otimes C$ as $(A, A)$-bimodules and right $C$-comodules.

(5) There exists an integral $x=\sum_{i=1}^{n} a_{i} \otimes c_{i}$ in $(A, C)_{\psi}$, such that the map $\phi: C^{*} \otimes A \rightarrow A \otimes C, \xi \otimes a \mapsto \sum_{i=1}^{n} a_{i} \psi\left(\xi \triangleright c_{i} \otimes a\right)$ is bijective.

Since $\mathbf{M}_{A}^{C}(\psi) \cong \mathbf{M}_{B \#_{\bar{\psi}} A}$ and $\operatorname{Hom}\left(C^{*},-\right) \cong-\otimes C$ (for $C$ is a finitely generated projective $k$-module), the first statement is the same as Proposition 3.2(1). Since $C^{*}$ is a right $C$-comodule via coproduct in $C$, both $C^{*} \otimes A, A \otimes C$ are objects in $\mathbf{M}_{A}^{C}(\psi)$ by Example 2.5 They are also right $B \#_{\bar{\psi}} A$ modules, via the category isomorphism described above. $C^{*} \otimes A$ is a left $A$-module via the multiplication in $B \#_{\bar{\psi}} A$, and $A \otimes C$ is a left $A$-module via $\mu \otimes C$. Thus the statements (3) and (4) make sense. The natural isomorphism $\operatorname{Hom}\left(A, C^{*}\right) \cong A \otimes C$ also implies that both these statements are equivalent to Proposition 3.2(3). For the same reason the $k$-module $\operatorname{Int}\left(B \#_{\bar{\psi}} A\right)$ is isomorphic to the $k$-module of integrals in $(A, C)_{\psi}$ and thus the statement (5) is just a reformulation of Proposition 3.2(4).

Remark 3.6. If $(A, C)_{\psi}$ is an entwining structure, $A$ is a faithfully flat $k$-module and $C$ is a projective $k$-module and if $-\otimes C$ is the left adjoint of the forgetful functor $\mathbf{M}_{A}^{C}(\psi) \rightarrow \mathbf{M}_{A}$, then $C$ is finitely generated. This follows from the fact that, in this case, for all $M \in \mathbf{M}_{A}, N \in \mathbf{M}_{A}^{C}(\psi), m \in M$, and $f \in \operatorname{Hom}_{A}^{C}(M \otimes C, N)$,

$$
\eta_{M, N}(f)(m)=\sum_{i=1}^{n} f\left(m \cdot a_{i} \otimes c_{i}\right)
$$

where $\eta_{M, N}: \operatorname{Hom}_{A}^{C}(M \otimes C, N) \rightarrow \operatorname{Hom}_{A}(M, N)$ is a natural isomorphism and $\sum_{i=1}^{n} a_{i} \otimes c_{i}=\eta_{A, A \otimes C}(A \otimes C)\left(1_{A}\right)$. This can be proven following the same arguments as in [6] Lemma 2.3, Theorem 2.4]. Therefore, in the case of an entwining structure associated to a Doi-Hopf datum $(A, C, H)$ (cf. Example 2.2), Proposition 3.5 is equivalent (up to left-right conventions, and to the assumption that $H$ has a bijective antipode) to [6, Theorem 2.4]. 
The following proposition generalises [6, Theorem 3.4] to any entwining structure.

Proposition 3.7. Let $(A, C)_{\psi}$ be an entwining structure and assume that $C$ is a projective $k$-module. Then the following statements are equivalent:

(1) There exists $e \in C$ such that $C^{*} \triangleright e=C$ and for all $a \in A, \psi(e \otimes a)=a \otimes e$.

(2) $C$ is a finitely generated $k$-module and there exists a right $C$-comodule isomorphism $\phi: C^{*} \rightarrow C$ such that the following diagram

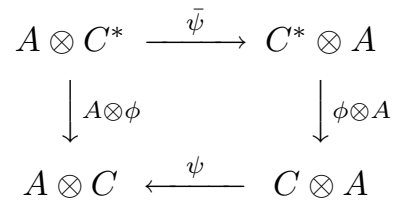

commutes $\left(C^{*}\right.$ is a right $C$-comodule via $\left.\xi_{(0)}\left\langle\xi_{(1)}, \xi^{\prime}\right\rangle=\xi^{\prime} \xi, \forall \xi, \xi^{\prime} \in C^{*}\right)$.

Furthermore, if $k$ is a field, then (1) and (2) are equivalent to:

(3) $C$ is finite dimensional and there exists a non-degenerate, bilinear and associative form $[-,-]: C^{*} \otimes C^{*} \rightarrow k$ such that the following diagram

$$
\begin{aligned}
& A \otimes C^{*} \otimes C^{*} \stackrel{\bar{\psi} \otimes C^{*}}{\longrightarrow} C^{*} \otimes A \otimes C^{*} \\
& \downarrow A \otimes[-,-] \quad \downarrow C^{*} \otimes \bar{\psi} \\
& A \quad \stackrel{[-,-] \otimes A}{\longleftarrow} C^{*} \otimes C^{*} \otimes A
\end{aligned}
$$

commutes.

(4) There exists $e \in C$ such that $e \triangleleft C^{*}=C$ and for all $a \in A, \psi(e \otimes a)=a \otimes e$.

Proof. (1) $\Rightarrow(2)$. Let $\Delta(e)=\sum_{i=1}^{n} c_{i} \otimes c_{i}^{\prime}$. Then $C=C^{*} \triangleright e \subseteq \sum_{i=1}^{n} k c_{i}$ and thus is finitely generated. Since $C$ is also projective, its dual $C^{*}$ is a finitely generated projective $k$-module. Consider $\phi: C^{*} \rightarrow C, \xi \mapsto \xi \triangleright e=e_{(1)}\left\langle e_{(2)}, \xi\right\rangle$. Clearly $\phi$ is a right $C$-comodule map. By assumption, $\phi$ is surjective and since both $C$ and $C^{*}$ are finitely generated projective $k$-modules of the same rank, we conclude that $\phi$ is an isomorphism.

Using the notation for $\bar{\psi}$ introduced in Section 2, we have for all $a \in A, \xi \in C^{*}$ :

$$
\begin{array}{rlrl}
\psi \circ(\phi \otimes A) \circ \bar{\psi}(a \otimes \xi) & =\left\langle e_{(2)}, \xi_{i}\right\rangle \psi\left(e_{(1)} \otimes a^{i}\right) & \\
& =\left\langle e_{(2)}^{\alpha}, \xi\right\rangle \psi\left(e_{(1)} \otimes a_{\alpha}\right) & & \text { (by the definition of } \bar{\psi}) \\
& =\left\langle e_{(2)}^{\alpha}, \xi\right\rangle a_{\alpha} \otimes e_{(1)}^{\alpha} & \text { (by Definition 2.1) } \\
& =a \otimes e_{(1)}\left\langle e_{(2)}, \xi\right\rangle & \quad(\text { for } \psi(e \otimes a)=a \otimes e) \\
& =a \otimes \phi(\xi) . &
\end{array}
$$

$(2) \Rightarrow(1)$. Given $\phi: C^{*} \rightarrow C$, define $e=\phi(\epsilon)$. Since $\phi$ is a right $C$-comodule map and $\epsilon$ is the unit in $C^{*}$ one easily finds that for all $\xi \in C, \phi(\xi)=\xi \triangleright e$. Thus $C^{*} \triangleright e=C$. The commutativity of the diagram in (2) together with the fact that, for all $a \in A, \bar{\psi}(a \otimes \epsilon)=\epsilon \otimes a$ now implies that $\psi(e \otimes a)=a \otimes e$, as required.

$(2) \Rightarrow(3)$. Given $\phi: C^{*} \rightarrow C$, define $[-,-]: C^{*} \otimes C^{*} \rightarrow k$, by $[-,-]:$ $\xi \otimes \xi^{\prime} \mapsto\left\langle\phi\left(\xi^{\prime}\right), \xi\right\rangle$. Since $\phi$ is right $C$-colinear, it is left $C^{*}$-linear and thus for all $\xi, \xi^{\prime}, \xi^{\prime \prime} \in C^{*}$

$$
\left[\xi \xi^{\prime}, \xi^{\prime \prime}\right]=\left\langle\phi\left(\xi^{\prime \prime}\right), \xi \xi^{\prime}\right\rangle=\left\langle\xi^{\prime} \triangleright \phi\left(\xi^{\prime \prime}\right), \xi\right\rangle=\left\langle\phi\left(\xi^{\prime} \xi^{\prime \prime}\right), \xi\right\rangle=\left[\xi, \xi^{\prime} \xi^{\prime \prime}\right],
$$

so that $[-,-]$ is associative. Since $\phi$ is onto, $C^{*} \ni \xi=0$ if and only if for all $\xi^{\prime} \in C^{*},\left[\xi, \xi^{\prime}\right]=\left\langle\phi\left(\xi^{\prime}\right), \xi\right\rangle=0$. Assume now that there exists $\xi \in C^{*}$ such that 
for all $\xi^{\prime} \in C^{*}$ we have $\left[\xi^{\prime}, \xi\right]=0$. Let $\left\{\xi_{n}, c_{n}\right\}_{n=1}^{N}$ be a dual basis in $C$. Then $0=\sum_{n=1}^{N}\left[\xi_{n}, \xi\right] c_{n}=\sum_{n=1}^{N}\left\langle\phi(\xi), \xi_{n}\right\rangle c_{n}=\phi(\xi)$, and thus $\xi=0$ since $\phi$ is bijective. Therefore the form $[-,-]$ is non-degenerate. Finally, take any $\xi, \xi^{\prime} \in C^{*}, a \in A$ and, using the same notation for $\bar{\psi}$ as before and the commutativity of the diagram in statement (2), compute $\left\langle\phi\left(\xi_{i}^{\prime}\right), \xi_{j}\right\rangle a^{j i}=\left\langle\phi\left(\xi_{i}^{\prime}\right)^{\alpha}, \xi\right\rangle a_{\alpha}^{i}=\left\langle\phi\left(\xi^{\prime}\right), \xi\right\rangle$. This implies the commutativity of the diagram in (3).

Now assume that $k$ is a field.

$(3) \Rightarrow(1)$. Given a non-degenerate, associative form $[-,-]$ on $C^{*}$, and a dual basis $\left\{\xi_{n}, c_{n}\right\}_{n=1}^{N}$ in $C$ define $e=\sum_{n=1}^{N}\left[\epsilon, \xi_{n}\right] c_{n}$. Then for all $\xi, \xi^{\prime} \in C^{*}$ one has $\left[\xi, \xi^{\prime}\right]=\left\langle e, \xi \xi^{\prime}\right\rangle=\left\langle\xi^{\prime} \triangleright e, \xi\right\rangle$. The non-degeneracy of $[-,-]$ implies that the map $C^{*} \rightarrow C, \xi \mapsto \xi \triangleright e$ is injective. Since both $C$ and $C^{*}$ have the same dimension, this map is also surjective. Now notice that for all $a \in A, \sum_{n=1}^{N} \psi\left(c_{n} \otimes a\right) \otimes \xi_{n}=$ $\sum_{n=1}^{N} a^{i} \otimes c_{n} \otimes \xi_{n i}$, where we use the same notation for $\bar{\psi}$ as before. Using this fact, as well as $\bar{\psi}(\epsilon \otimes a)=a \otimes \epsilon$ and the commutativity of the diagram in (3) one computes

$$
\psi(e \otimes a)=\sum_{n=1}^{N}\left[\epsilon, \xi_{n}\right] \psi\left(c_{n} \otimes a\right)=\sum_{n=1}^{N}\left[\epsilon_{i}, \xi_{n j}\right] a^{i j} \otimes c_{n}=\sum_{n=1}^{N} a \otimes\left[\epsilon, \xi_{n}\right] c_{n}=a \otimes e .
$$

$(4) \Rightarrow(1)$. The similar argument as in the proof of the first implication shows that $C$ is finite dimensional. Since $e \triangleleft C^{*}=C$, we have for all $\xi \in C,\langle C, \xi\rangle=$ $\left\langle e \triangleleft C^{*}, \xi\right\rangle=\left\langle\xi \triangleright e, C^{*}\right\rangle$. Therefore, $\xi \triangleright e=0$ implies $\xi=0$ so that the map $\xi \mapsto \xi \triangleright e$ is injective. Since both $C$ and $C^{*}$ are of the same finite dimension, we conclude that $C^{*} \triangleright e=C$. Using a similar argument one shows the implication $(1) \Rightarrow(4)$.

Statement (1) of Proposition 3.7 takes a very simple form in the case of the canonical entwining structure $(A, C)_{\psi}$ associated to a $C$-Galois extension $A(B)^{C}$. In this case $e \in C$ has the property that, for all $a \in A, \psi(e \otimes a)=a \otimes e$, if and only if for all $a \in A, a \tau(e)=\tau(e) a$, where $\tau(c)=\operatorname{can}^{-1}(1 \otimes c)$. This is a simple consequence of the definition of the canonical entwining structure in Example 2.3

\section{MASCHKE-TYPE THEOREMS}

The classical Maschke's Theorem states that a group ring of a finite group is semisimple if and only if the characteristic of the field does not divide the order of the group. Several generalisations of Maschke's theorem to Hopf algebras and comodule algebras are known [12, [8], 1]. In 7] a Maschke-type theorem was formulated for Doi-Hopf modules. Following [7] we define

Definition 4.1. Let $(A, C)_{\psi}$ be an entwining structure. A $k$-module map $\phi: C \rightarrow$ $C^{*} \otimes A$ is called a normalised integral map in $(A, C)_{\psi}$ if

(1) $\mu \circ\left(A \otimes \mathrm{ev}_{C} \otimes A\right) \circ(\psi \otimes \phi) \circ(C \otimes \psi)=\left(\mathrm{ev}_{C} \otimes \mu\right) \circ(C \otimes \phi \otimes A)$,

(2) $\left(\mathrm{ev}_{C} \otimes A \otimes C\right) \circ(C \otimes \phi \otimes C) \circ(C \otimes \Delta)=\psi \circ\left(C \otimes \mathrm{ev}_{C} \otimes A\right) \circ(\Delta \otimes \phi)$,

(3) $\left(\mathrm{ev}_{C} \otimes A\right) \circ(C \otimes \phi) \circ \Delta=1_{A} \circ \epsilon$.

If $(A, C)_{\psi}$ is a factorisable entwining structure, then conditions (1) and (2) in Definition 4.1 state that $\phi$ is an $\left(A, C^{* o p} \#{ }_{\bar{\psi}} A\right)$-bimodule map. Condition (3) is a normalisation condition. In particular, for an entwining structure of Example 2.2 Definition 4.1] is equivalent to [7] Definition 2.1] (up to the left-right conventions and the bijectivity of an antipode). The following generalises [7, Theorem 2.5]. 
Theorem 4.2. If there exists a normalised integral map in $(A, C)_{\psi}$, then a morphism in $\mathbf{M}_{A}^{C}(\psi)$ which has a section (resp. retraction) in $\mathbf{M}_{A}$ has a section (resp. retraction) in $\mathbf{M}_{A}^{C}(\psi)$.

Lemma 4.3. Let $M, N \in \mathbf{M}_{A}^{C}(\psi), g \in \operatorname{Hom}_{A}(M, N)$ and let $\phi$ be a normalised integral map in $(A, C)_{\psi}$. Then $\tilde{g}: M \rightarrow N, \tilde{g}=\rho_{N} \circ\left(N \otimes \mathrm{ev}_{C} \otimes A\right) \circ\left(\rho^{N} \circ g \otimes \phi\right) \circ \rho^{M}$ is a morphism in $\mathbf{M}_{A}^{C}(\psi)$.

Proof. We use the Sweedler-like notation $\phi(c)=c^{(1)} \otimes c^{(2)} \in C^{*} \otimes A$ (summation understood), for any $c \in C$. Then $\tilde{g}(m)=g\left(m_{(0)}\right)_{(0)} \cdot m_{(1)}{ }^{(2)}\left\langle g\left(m_{(0)}\right)_{(1)}, m_{(1)}{ }^{(1)}\right\rangle$, for all $m \in M$. To show that $\tilde{g}$ is right $A$-linear, take any $m \in M, a \in A$ and compute

$$
\begin{array}{rlrl}
\tilde{g}(m \cdot a) & =g\left((m \cdot a)_{(0)}\right)_{(0)} \cdot(m \cdot a)_{(1)}{ }^{(2)}\left\langle g\left((m \cdot a)_{(0)}\right)_{(1)},(m \cdot a)_{(1)}{ }^{(1)}\right\rangle \\
& =g\left(m_{(0)} \cdot a_{\alpha}\right)_{(0)} \cdot m_{(1)}{ }^{\alpha(2)}\left\langle g\left(m_{(0)} \cdot a_{\alpha}\right)_{(1)}, m_{(1)}{ }^{\alpha(1)}\right\rangle & \left(M \in \mathbf{M}_{A}^{C}(\psi)\right) \\
& =\left(g\left(m_{(0)}\right) \cdot a_{\alpha}\right)_{(0)} \cdot m_{(1)}{ }^{\alpha(2)}\left\langle\left(g\left(m_{(0)}\right) \cdot a_{\alpha}\right)_{(1)}, m_{(1)}{ }^{\alpha(1)}\right\rangle & (g \text { is } A \text {-linear }) \\
& =g\left(m_{(0)}\right)_{(0)} \cdot a_{\alpha \beta} m_{(1)}{ }^{\alpha(2)}\left\langle g\left(m_{(0)}\right)_{(1)}{ }^{\beta}, m_{(1)}{ }^{\alpha(1)}\right\rangle & & \left(N \in \mathbf{M}_{A}^{C}(\psi)\right) \\
& =g\left(m_{(0)}\right)_{(0)} \cdot m_{(1)}{ }^{(2)} a\left\langle g\left(m_{(0)}\right)_{(1)}, m_{(1)}{ }^{(1)}\right\rangle & & (\text { Def. 4.1(1)) } \\
& =\tilde{g}(m) \cdot a . &
\end{array}
$$

Next, for all $m \in M$, we have

$$
\begin{aligned}
\rho^{N}(\tilde{g}(m)) & =\left(g\left(m_{(0)}\right) \cdot m_{(1)}{ }^{(2)}\right)_{(0)}\left\langle g\left(m_{(0)}\right)_{(1)}, m_{(1)}{ }^{(1)}\right\rangle \otimes\left(g\left(m_{(0)}\right) \cdot m_{(1)}{ }^{(2)}\right)_{(1)} \\
& =g\left(m_{(0)}\right)_{(0)} \cdot m_{(1)}{ }^{(2)}{ }_{\alpha}\left\langle g\left(m_{(0)}\right)_{(2)}, m_{(1)}{ }^{(1)}\right\rangle \otimes g\left(m_{(0)}\right)_{(1)}{ }^{\alpha} \\
& =g\left(m_{(0)}\right)_{(0)} \cdot m_{(1)}{ }^{(2)}\left\langle g\left(m_{(0)}\right)_{(1)}, m_{(1)}{ }^{(1)}\right\rangle \otimes m_{(2)} \\
& =\tilde{g}\left(m_{(0)}\right) \otimes m_{(1)},
\end{aligned}
$$

where we used that $N \in \mathbf{M}_{A}^{C}(\psi)$ to derive the second equality.

Proof of Theorem 4.2. Let $M, N \in \mathbf{M}_{A}^{C}(\psi)$, and assume that $f \in \operatorname{Hom}_{A}^{C}(N, M)$ has a section $g \in \operatorname{Hom}_{A}(M, N)$. Let $\tilde{g} \in \operatorname{Hom}_{A}^{C}(M, N)$ be as in Lemma 4.3. Then for all $m \in M$,

$$
\begin{aligned}
f \circ \tilde{g}(m) & =f\left(g\left(m_{(0)}\right)_{(0)} \cdot m_{(1)}{ }^{(2)}\right)\left\langle g\left(m_{(0)}\right)_{(1)}, m_{(1)}{ }^{(1)}\right\rangle \\
& =f\left(g\left(m_{(0)}\right)\right)_{(0)} m_{(1)}{ }^{(2)}\left\langle f\left(g\left(m_{(0)}\right)\right)_{(1)}, m_{(1)}{ }^{(1)}\right\rangle \quad\left(f \in \operatorname{Hom}_{A}^{C}(N, M)\right) \\
& =m_{(0)} \cdot m_{(2)}{ }^{(2)}\left\langle m_{(1)}, m_{(2)}{ }^{(1)}\right\rangle=m \quad(g \text { is a section of } f, \text { Def. 4.1(3)). }
\end{aligned}
$$

Similar computation shows that if $g$ is a retraction of $f$, then so is $\tilde{g}$.

Corollary 4.4. If there is a normalised integral map in $(A, C)_{\psi}$, then

(1) Every object in $\mathbf{M}_{A}^{C}(\psi)$ which is semisimple as an object in $\mathbf{M}_{A}$ is semisimple as an object in $\mathbf{M}_{A}^{C}(\psi)$.

(2) Every object in $\mathbf{M}_{A}^{C}(\psi)$ which is projective (resp. injective) as a right $A$ module is a projective (resp. injective) object in $\mathbf{M}_{A}^{C}(\psi)$.

(3) If $C$ is a flat $k$-module, then $M \in \mathbf{M}_{A}^{C}(\psi)$ is projective as a right $A$-module if and only if there exists $V \in \mathbf{M}^{C}$ such that $M$ is a direct summand of $V \otimes A$ in $\mathbf{M}_{A}^{C}(\psi)(V \otimes A$ is an entwined module by Example 2.5(2)).

Proof. The first statement follows immediately from Theorem 4.2 Assertions (2) and (3) can be proven by the same method as 7, Corollary 2.9]. In particular to prove (2) it is useful to observe that if $f$ is a morphism in $\mathbf{M}_{A}^{C}(\psi)$, then $\tilde{f}=f$, where 
$\tilde{f}$ is constructed in Lemma 4.3. To prove (3) one uses that the forgetful functor $\mathbf{M}_{A}^{C}(\psi) \rightarrow \mathbf{M}_{A}$ is the left adjoint of the exact functor $-\otimes C: \mathbf{M}_{A} \rightarrow \mathbf{M}_{A}^{C}(\psi)$ (cf. [3, Section 3]).

We can dualise the above construction to derive the dual version of Theorem 4.2

Definition 4.5. Let $(A, C)_{\psi}$ be an entwining structure with $A$ a finitely generated projective $k$-module. Let $\operatorname{coev}_{A}: k \rightarrow A \otimes A^{*}$ be a coevaluation map, i.e., $\operatorname{coev}_{A}$ : $\kappa \mapsto \kappa \sum_{i \in I} a_{i} \otimes a_{i}^{*}$, where $\left\{a_{i}, a_{i}^{*}\right\}_{i \in I}$ is a dual basis in $A$. A $k$-module map $\phi: A^{*} \otimes C \rightarrow A$ is called a normalised cointegral map in $(A, C)_{\psi}$ if

(1) $(A \otimes \psi) \circ(\psi \otimes \phi) \circ\left(C \otimes \operatorname{coev}_{A} \otimes C\right) \circ \Delta=(A \otimes \phi \otimes C) \circ\left(\operatorname{coev}_{A} \otimes \Delta\right)$,

(2) $(A \otimes \mu) \circ(A \otimes \phi \otimes A) \circ\left(\operatorname{coev}_{A} \otimes C \otimes A\right)=(\mu \otimes \phi) \circ\left(A \otimes \operatorname{coev}_{A} \otimes C\right) \circ \psi$,

(3) $\mu \circ(A \otimes \phi) \circ\left(\operatorname{coev}_{A} \otimes C\right)=1_{A} \circ \epsilon$.

Conditions (1) and (2) in Definition 4.5 can be understood as follows. $A^{*}$ is a right $A$-module with the action $\left\langle a^{\prime}, a^{*} \cdot a\right\rangle=\left\langle a a^{\prime}, a^{*}\right\rangle$, for any $a, a^{\prime} \in A, a^{*} \in A^{*}$. Thus $A^{*} \otimes C$ is an entwined module as in Example 2.5(1). Furthermore, $\psi$ induces the map $\hat{\psi}: A^{*} \otimes C \rightarrow C \otimes A^{*}, a^{*} \otimes c \mapsto \sum_{i \in I} c^{\alpha} \otimes a_{i}^{*}\left\langle a_{i \alpha}, a^{*}\right\rangle$. Using this map one defines a left coaction of $C$ on $A^{*} \otimes C$ as $(\hat{\psi} \otimes C) \circ\left(A^{*} \otimes \Delta\right)$. The $k$-module $C \otimes A$ has a right $A$ module and right $C$-comodule structure as in Example 2.5 (2), and a left $C$-comodule structure, $\Delta \otimes C$. Conditions (1) and (2) of Definition 4.5 are equivalent to the existence of $\tilde{\phi} \in{ }^{C} \operatorname{Hom}_{A}^{C}\left(A^{*} \otimes C, C \otimes A\right)($ then $\phi=(\epsilon \otimes A) \circ \tilde{\phi})$.

Theorem 4.6. If there exists a normalised cointegral map in $(A, C)_{\psi}$, then any morphism in $\mathbf{M}_{A}^{C}(\psi)$ which has a section (resp. retraction) in $\mathbf{M}^{C}$ has a section (resp. retraction) in $\mathbf{M}_{A}^{C}(\psi)$.

Lemma 4.7. Let $M, N \in \mathbf{M}_{A}^{C}(\psi), g \in \operatorname{Hom}^{C}(M, N)$ and let $\phi$ be a normalised cointegral map in $(A, C)_{\psi}$. Then $\tilde{g}: M \rightarrow N$,

$$
\tilde{g}=\rho_{N} \circ\left(g \circ \rho_{M} \otimes \phi\right) \circ\left(M \otimes \operatorname{coev}_{A} \otimes C\right) \circ \rho^{M}
$$

is a morphism in $\mathbf{M}_{A}^{C}(\psi)$.

Proof. Dual to the proof of Lemma4.3.

Proof of Theorem 4.6. Let $M, N \in \mathbf{M}_{A}^{C}(\psi)$, and assume that $f \in \operatorname{Hom}_{A}^{C}(N, M)$ has a section $g \in \operatorname{Hom}^{C}(M, N)$. Let $\tilde{g} \in \operatorname{Hom}_{A}^{C}(N, M)$ be as in Lemma 4.7. Explicitly, for all $m \in M, \tilde{g}(m)=\sum_{i \in I} g\left(m_{(0)} \cdot a_{i}\right) \cdot \phi\left(a_{i}^{*} \otimes m_{(1)}\right)$, where $\left\{a_{i}, a_{i}^{*}\right\}_{i \in I}$ is a dual basis in $A$. Thus

$$
\begin{aligned}
f \circ \tilde{g}(m) & =\sum_{i \in I} f\left(g\left(m_{(0)} \cdot a_{i}\right) \cdot \phi\left(a_{i}^{*} \otimes m_{(1)}\right)\right) \\
& =\sum_{i \in I} f\left(g\left(m_{(0)} \cdot a_{i}\right)\right) \cdot \phi\left(a_{i}^{*} \otimes m_{(1)}\right) \quad(f \text { is right } A \text {-linear }) \\
& =\sum_{i \in I} m_{(0)} \cdot a_{i} \phi\left(a_{i}^{*} \otimes m_{(1)}\right)=m \quad(g \text { is a section of } f, \text { Def. } 4.5) .
\end{aligned}
$$

Similar computation shows that if $g$ is a retraction of $f$, then so is $\tilde{g}$.

Theorem 4.6 implies that if there is a normalised cointegral map in $(A, C)_{\psi}$, then every object in $\mathbf{M}_{A}^{C}(\psi)$ which is semisimple as an object in $\mathbf{M}^{C}$ is semisimple as an object in $\mathbf{M}_{A}^{C}(\psi)$. Furthermore every object in $\mathbf{M}_{A}^{C}(\psi)$ which is a projective (resp. injective) object in $\mathbf{M}^{C}$ is projective (resp. injective) in $\mathbf{M}_{A}^{C}(\psi)$. 


\section{REFERENCES}

[1] R.J. Blattner and S. Montgomery. Crossed products and Galois extensions of Hopf algebras. Pacific. J. Math., 137:37-54, 1989. MR 90a:16007

[2] N. Bourbaki. Commutative Algebra Addison-Wesley, Reading, 1972. MR 50:12997

[3] T. Brzeziński. On modules associated to coalgebra-Galois extensions. Preprint, qalg/9712023, 1997. J. Algebra, 215:290-317, 1999. CMP 99:11

[4] T. Brzeziński and P.M. Hajac. Coalgebra extensions and algebra coextensions of Galois type. Preprint, q-alg/9708010, 1997. Commun. Algebra, 27:1347-1367, 199. CMP 99:09

[5] T. Brzeziński and S. Majid. Coalgebra bundles. Commun. Math. Phys., 191:467-492, 1998. q-alg/9602022, 1996. CMP 98:08

[6] S. Caenepeel, G. Militaru and S. Zhu. Doi-Hopf modules, Yetter-Drinfel'd modules and Frobenius type properties. Trans. Amer. Math. Soc. , 349:4311-4342, 1997. MR 98i:16035

[7] S. Caenepeel, G. Militaru and S. Zhu. A Maschke-type Theorem for Doi-Hopf modules and Applications. J. Algebra, 187:388-412, 1997. MR 98e:16034

[8] Y. Doi. Hopf extensions of algebras and Maschke-type theorems. Israel J. Math. , 72:99-108, 1990. MR 92b:16078

[9] Y. Doi. Unifying Hopf modules. J. Algebra, 153:373-385, 1992. MR 94c:16048

[10] F. Kasch. Projektive Frobenius-Erweiterungen. Sitzungsber. Heidelberger Akad. Wiss. Math.Natur. Kl. , 89-109, 1960/61. MR 24:A1932

[11] M. Koppinen. Variations on the smash product with applications to group-graded rings. $J$. Pure Appl. Alg. , 104:61-80, 1994. MR 96j:16028

[12] R.G. Larson and M.E. Sweedler. An associative orthogonal bilinear form for Hopf algebras. Amer. J. Math. , 91:75-93, 1969. MR 39:1523

[13] S. Majid. Foundations of Quantum Group Theory. Cambridge University Press, 1995. MR 97g:17016

[14] C. Menini and C. Năstăsescu. When are induction and coinduction functors isomorphic? Bull. Belg. Math. Soc. 1:521-558, 1994. [MR 96a:16041

[15] T. Nakayama and T. Tsuzuku. On Frobenius extensions I. Nagoya. Math. J. 17:89-110, 1960. MR 23:A1687

Department of Mathematics, University of York, Heslington, York YO10 5DD, United KINGDOM

E-mail address: tb10@york.ac.uk

$U R L: \mathrm{http} / /$ www. york.ac.uk/ ${ }^{\mathrm{tb} 10}$

Department of Theoretical Physics, University of Łódź, Pomorska 149/153, 90-236

ŁÓDŹ, Poland 